\title{
Noise Reduction in Rhythmic and Multitrial Biosignals With Applications to Event-Related Potentials
}

\author{
Patrick Celka*, Khoa N. Le, Member, IEEE, and Timothy R. H. Cutmore
}

\begin{abstract}
A new noise reduction algorithm is presented for signals displaying repeated patterns or multiple trials. Each pattern is stored in a matrix, forming a set of events, which is termed multievent signal. Each event is considered as an affine transform of a basic template signal that allows for time scaling and shifting. Wavelet transforms, decimated and undecimated, are applied to each event. Noise reduction on the set of coefficients of the transformed events is applied using either wavelet denoising or principal component analysis (PCA) noise reduction methodologies. The method does not require any manual selection of coefficients. Nonstationary multievent synthetic signals are employed to demonstrate the performance of the method using normalized mean square error against classical wavelet and PCA based algorithms. The new method shows a significant improvement in low SNRs (typically $<0 \mathrm{~dB}$ ). On the experimental side, evoked potentials in a visual oddball paradigm are used. The reduced-noise visual oddball event-related potentials reveal gradual changes in morphology from trial to trial (especially for N1-P2 and N2-P3 waves at $F z$ ), which can be hypothetically linked to attention or decision processes. The new noise reduction method is, thus, shown to be particularly suited for recovering single-event features in nonstationary low SNR multievent contexts.
\end{abstract}

Index Terms-Event-related potentials (ERPs), noise reduction, principal component analysis (PCA), rhythmic patterns, visual oddball paradigm, wavelet.

\section{INTRODUCTION}

I N BIOMEDICAL signal processing, translational shifts and embedded-noise in signals are very common. Many different approaches for noise reduction of biological signals have been extensively developed. Among the most noisy biosignals are probably electroencephalograms (EEGs) and event-related brain potentials for which the SNR is often well below $0 \mathrm{~dB}$. For this reason, it is interesting to focus on the denoising methods that have been developed for such signals. We can classify the biosignal noise reduction methods into two classes: 1) those using nonlinear time series techniques [1]-[3] and 2) those based

Manuscript received April 24, 2007; revised December 1, 2007. This work was supported by Griffith University under Griffith University Research Grant: Explaining the Flash-Lag Illusion Using Evoked Potentials. Asterisk indicates corresponding author.

${ }^{*}$ P. Celka was with the Griffith School of Engineering, Griffith University, Southport Queensland 4215, Australia. He is now with the Lama Tzong Khapa Institute, I-56040 Pomaia (PI), Italy (e-mail: p.celka@gmail.com).

K. N. Le is with the Griffith School of Engineering, Griffith University, Southport Queensland 4215, Australia (e-mail: k.le@ griffith.edu.au).

T. R. H. Cutmore is with the School of Psychology, Griffith University, Southport Queensland 4215, Australia (e-mail: t.cutmore@griffith.edu.au).

Color versions of one or more of the figures in this paper are available online at http://ieeexplore.ieee.org.

Digital Object Identifier 10.1109/TBME.2008.919851 on linear transforms [4]-[11]. Biological signals are often locally linear (on a relatively small time window); accordingly, two main denoising approaches have been developed based on: 1) principal component analysis (PCA) [12], also called singular value decomposition technique and 2) wavelet transforms [13]. One of the important features of an efficient noise reduction is to restore the time-varying information hidden in the noisy signals. Another feature of these linear methods is their low complexity as compared to nonlinear ones, which thus require more processing and memory resources [14]. For these reasons, PCA and wavelet transforms are the most popular, and thus, are used in this study.

Biosignals often present rhythmical patterns that may be additionally used to reduce undesirable fluctuations found in electrocardiograms, photoplethysmograms, and respiratograms. In some other situations such as event-related potential (ERP) studies, multiple trials may be recorded. The term event is adopted for either a trial or a pattern of a rhythm, and the set of all the events is called a multievent. In such a context, researchers have developed strategies to reduce the noise in these multievent signals using statistical averaging: averaging across all the events; assuming that the random fluctuations on the events were additive with respect to the information signal, independent from event to event and zero-mean. This statistical averaging also suffers from other drawbacks such as: 1) the events are assumed to be stationary and 2) the output of the method is eventually a single noise reduced event, which means that we have lost all the information contained in the single events.

Recent works have started to address these issues more closely [9], [15]: Browne and Cutmore [15] used a multievent technique to remove unlikely artifacts in event-related signals where the subspace decomposition was the decimated (subsampled) discrete wavelet transform (DWT). Quiroga [9] and Celka and Gysels [16] used the DWT on each event using the a posteriori knowledge of the statistical average [9], [16]. It is the aim of this study to propose a new denoising strategy for multievent signals. Our proposed approach unfolds in three steps: 1) we decompose each event using a wavelet transform, and we collect all the wavelet coefficients of all events for each scale; 2) denoising is then performed on these wavelet coefficients across all events for each scale using either a PCA [14], [17] or a wavelet denoising method, i.e., we apply either a PCA decomposition followed by a selection of the best coefficients with a preselected dimension for the signal space (the space that is supposed to contain the least possible noise), or a wavelet transform followed by a selection of the best coefficients using 
the so-called stein unbiased estimate of risk (SURE) introduced by Donoho and Johnstone [18]; and 3) we finally perform the inverse wavelet transform to the denoised wavelet coefficients corresponding to each event. It is important to note the difference between our approach that uses all the wavelet coefficients of the all the events to perform the denoising in step 2, from other methods that denoise one event at a time. The PCA and waveletbased denoising techniques in step 2 are used for performance comparison purposes only.

Step 1 uses a wavelet transform for the subspace decomposition, thus acting as a filter bank. The continuous and DWTs are useful time-scale analysis tools [19], [20], which can thus be used for subspace decomposition. One of the few flaws of the DWT is that it is time-shift variant, which means that time shifting the signals does not results in time shifting their wavelet coefficients. In particular, the number of nonzero DWT coefficients for the translated case is sometimes larger than that for the nontranslated case. This means that the DWT would be less effective when the input signal is translated because more information-carrying DWT coefficients are lost when employing a coefficient shrinking noise reduction scheme [21]. Coifman and Donoho proposed a translation-invariant method [22], which was later used by Lang et al. [21], Pesquet et al. [23], and Cambolle and Lucier [24]. To remedy the translationalvariant property of the DWT, the undecimated wavelet transform (UDWT) is employed. The main difference between the UDWT and DWT is that decimators are removed from the filterbank structure in the former transform. As such, the UDWT is redundant and is also known as the redundant DWT [25].

The paper is organized as follows. Section II describes our affine transform assumption of the events. Section III elaborates the proposed strategy that uses a wavelet transform on a multievent set prior to the actual denoising on the set of resulting wavelet coefficients. Section IV describes two other denoising techniques based on a PCA transform in state space and a wavelet denoising that are further used for comparison with our new technique. Section V explains the performance measures that we have used to validate our strategy. Section VI presents the synthetic data and the experimental protocol that we have used, both in the context of ERP signals. Section VII shows the results for both the type of data and discusses them. Finally, Section VIII concludes the paper with possible recommendations and future research directions.

\section{AfFine Transform Assumption}

Suppose that we have a finite set of $N$ discrete time events $x^{(k)}(n)$ for $k=1, \ldots, N$, where $1 \leq n \leq L$ is the discrete time variable. These events are either individual trials or repetitive patterns extracted from a rhythmical signal with a time trigger event. The events have a random component $\eta^{(k)}(n)$, which linearly combines with the signal of interest $s^{(k)}(n)$. Each $s^{(k)}(n)$ is supposed to be an affine transform of a basic pattern $s_{b}(n)$

$$
s^{(k)}(n)=s_{b}\left(\frac{n-T}{a}\right)
$$

where $T$ and $a>0$ are the translation and time-scaling parameters of the affine transform, respectively. Once the individual events are transformed using a wavelet transform, the question is how do the $N$ sets of coefficients fluctuate across all scales and time? Now, if the events are affine transforms of a template and if UDWT (time shift-invariant) is used, it will be possible to find a regular pattern across the $N$ events' wavelet coefficients. The advantage of having a regular pattern across the $N$ events' wavelet coefficients is that the PCA-based denoising method used in step 2 will be the most effective (as further validated by our performance measures on synthetic data). This can be understood in a simple way: with $N$ events at disposal, we have more information about the psychophysical processes taking place. Each wavelet coefficient is a random process with a given density function. The goal of the denoising or noise reduction, as we like to call it, is to minimize the uncertainty around each wavelet coefficient. Because each wavelet coefficient is supposed to change rather slowly (as explained in the Affine Transform Section) across events, we can then use these coefficients to form a stochastic process containing a signal space and a noise space. Thus, performing denoising on these coefficients eventually decreases their uncertainty, thus reducing the variance on each of them.

One drawback of the UDWT is its computational complexity as compared to the DWT. We, therefore, wanted to test the performance of the DWT (which is not time-shift invariant) against the one of the UDWT.

\section{ALgORITHM}

The key difference between our proposed noise reduction algorithm and other works is that the actual denoising is performed on the sets of the wavelet coefficients computed and grouped from all the events. Other denoising techniques consider event by event with eventually the use of a posteriori or a priori knowledge to improve the localization of information-bearing transformed coefficients (transforms can be Fourier, wavelet, PCA, etc.) [9], [16]. Our method is implemented as follows.

\section{A. Step 1: DWT or UDWT}

The goal of performing a signal transform in this first step is to have a more compact representation of the information and a primary step toward the separation of the signal space (the information we want) and the noise space (the information that we do not want). The goal in this first step is to separate the signal from the noise in the dual domain of the user's choice. We use a dyadic wavelet transform decomposition, either DWT or UDWT, of each event $k$ to obtain the following wavelet coefficient vector

$$
\overline{\mathbf{X}}^{(k)}=\mathcal{W} \mathbf{x}^{(k)}
$$

where $\mathcal{W}$ represents the DWT or UDWT. We have used the vector notations for the event signals: $\left(\mathbf{x}^{(k)}\right)^{T}=$ $\left(x^{(k)}(1), \ldots, x^{(k)}(L)\right)$ and the wavelet coefficients $\left(\overline{\mathbf{X}}^{(k)}\right)^{T}=$ $\left(\left(\mathbf{X}_{0}^{(k)}\right)^{T}, \ldots,\left(\mathbf{X}_{S c}^{(k)}\right)^{T}\right)$, where $S c$ is the number of scales used in the wavelet transform.

If DWT is used, $\mathcal{W}$ can be implemented as an $L \times L$ wavelet transform matrix (possibly orthogonal) and can be implemented using fast algorithms [26], and we have $\sum_{j} L_{j}=L$, where $L_{j}$ are the number of coefficients at scale $j$, and we have the 

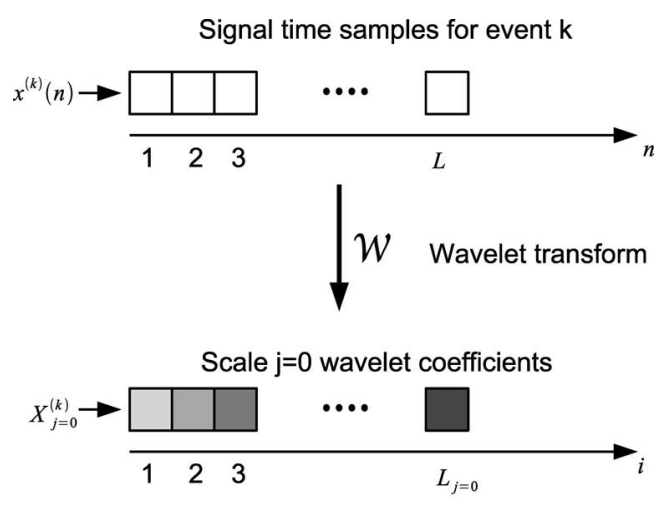

Scale $\mathrm{j}=1$ wavelet coefficients

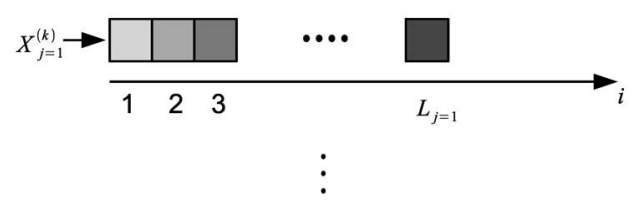

Scale $\mathrm{j}=$ Sc wavelet coefficients

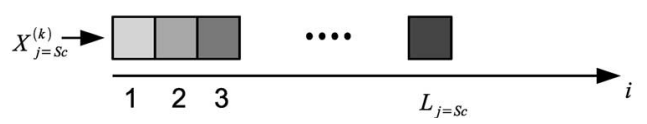

Fig. 1. Wavelet transform of a single event signal $\mathbf{x}^{(k)}$ into a set of wavelet coefficient vectors $\mathbf{X}_{j}^{(k)}$ for $j=0, \ldots, S c$. Each box corresponds to a sample of $\mathbf{x}^{(k)}$ or a wavelet coefficient $X_{j}^{(k)}(i)$. For $j=0$, we have the approximation coefficients (low pass) and $j \geq 0$ the details coefficients (high pass).

relationship between the number of scales and the number of signal samples: $S c=\log _{2}(L)-1$.

If UDWT is used, $\mathcal{W}$ is implemented using a nonsquare matrix form (thus, nonorthogonal) of size $(S c+1) L \times L$ and we have $\sum_{j} L_{j}=(S c+1) L$. In this case, the matrix $\mathcal{W}$ is not invertible, while a left-inverse matrix $\mathcal{W}_{(L)}^{-1}$ can be computed. Applying the UDWT to the signal $\mathbf{x}^{(k)}$ yields $K=2^{S c}$ different transformed signals $\overline{\mathbf{X}}_{l}^{(k)}$ for $l=1, \ldots, K$, resulting in, at least, $K$ different ways of reconstructing the signals $\mathbf{x}_{l}^{(k)}$. The more common approach to solve this ambiguity is to perform averaging of all the reconstructed signals $\mathbf{x}_{l}^{(k)}$ over $l$. This is equivalent to using the pseudoinverse of $\mathcal{W}_{(L)}^{-1}$ for the reconstruction. For either the DWT or UDWT case, we call the (left pseudo)-inverse wavelet matrix $\mathcal{W}^{-1}$.

Recalling that we have $k$ event signals, the wavelet coefficients $\left(\mathbf{X}_{j}^{(k)}\right)^{T}=\left(X_{j}^{(k)}(1), \ldots, X_{j}^{(k)}\left(L_{j}\right)\right)$ can then be considered as signals, which are functions of the event number $k$ for each scale $j$ and time $i$, i.e., $\Xi_{i j}(k)=X_{j}^{(k)}(i)$. In other words, we consider the wavelet coefficients across all events for each scale $j$ and time $i$ fixed. Figs. 1 and 2 show the wavelet decomposition of one event $k$, and the procedure for building the signals $\Xi_{i j}(k)$. The events in $\Xi_{i j}(k)$ are arranged consecutively from $k=1$ to $k=N$ as they naturally appear in the time. So, for instance, if the multievent signal is made up from different trials, even if each trial appears randomly in the time, they are arranged consecutively by order of appearance.

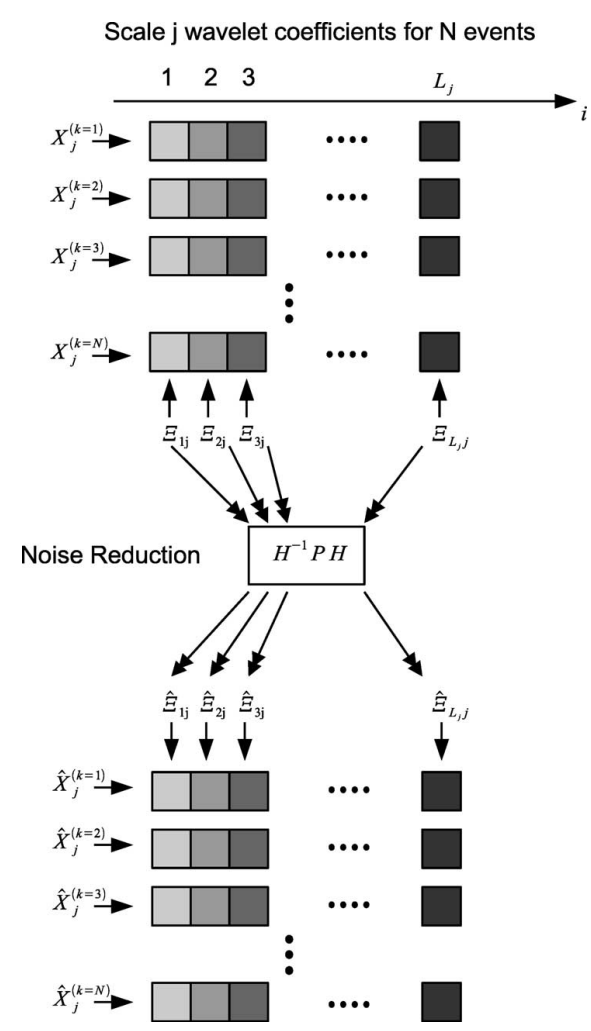

Fig. 2. Wavelet decomposition of all the $k$ events $\mathbf{x}^{(k)}$ is then used to construct the signals $\Xi_{i j}(k)=X_{j}^{(k)}(i)$. This figure shows the procedure to construct the signals $\Xi_{i j}(k)$ for the scale $j$, followed by the noise reduction using the operator $\mathbf{H}^{-1} P \mathbf{H}$ yielding a new set of coefficients $\hat{\boldsymbol{\Xi}}_{i j}$.

At this stage, the signal $\Xi_{i j}^{T}=\left(\Xi_{i j}(1), \ldots, \Xi_{i j}(N)\right)$ contains a random part, which should be reduced, and a more deterministic part, which should be retained. The random part in the wavelet coefficients originates from the noise contained in the signals $\mathbf{x}^{(k)}$, which, in turn, can be either due to the measurement device (exogenous) or unknowable internal sources (endogenous). Either way, these temporal noise sources are gathered in a single signal $\eta^{(k)}(n)$ as in (9), which generate the randomness in $\boldsymbol{\Xi}_{i j}$.

It should be noted that a standard noise reduction technique would shrink or select a subset of the wavelet coefficients $\mathbf{X}_{j}^{(k)}$ for each event $k$ individually, and then, proceed with the inverse wavelet transform $\mathcal{W}^{-1}$ to obtain a reduced noise event. Our method takes on a further step in making use of the availability of the $k$ events to denoise the signals $\Xi_{i j}(k)$ using either the SURE wavelet method or a PCA-based technique.

\section{B. Step 2: Noise Reduction}

The second stage of the algorithm is thus to reduce random fluctuations in $\boldsymbol{\Xi}_{i j}$ : the actual denoising. To do that, two classical noise reduction approaches are employed: 1) based on the PCA [17], [14] and 2) based on the wavelet SURE algorithm [18]. Both approaches can be formulated using the following matrix notation

$$
\begin{array}{r}
\hat{\mathbf{\Xi}}_{i j}=\left(\mathbf{H}^{-1} \mathbf{P} \mathbf{H}\right) \boldsymbol{\Xi}_{i j}, \quad \text { for } \quad i=1, \ldots, L_{j} ; \\
\quad \text { and } \quad j=0, \ldots, S c
\end{array}
$$


where $\mathrm{H}$ is the matrix that is used to perform either a PCA or a DWT, and $\mathbf{P}$ is a diagonal matrix used to select/shrink the coefficients $\mathbf{H} \mathbf{\Xi}_{i j}$, which is the core of the noise reduction. The operator $\mathbf{H}$ can be any subspace decomposition method. It should be noted that we do not directly select or threshold the coefficients $\boldsymbol{\Xi}_{i j}$ to perform the denoising, which would results from imposing $\mathbf{H}=I$ in (3), but a subspace decomposition of $\boldsymbol{\Xi}_{i j}$, expressed by $\mathbf{H}$, is imposed. Fig. 2 illustrates the operations performed in Step 2. We summarize these four different Methods in the following list

PCA: The DWT is used for Step 1 and PCA is used for Step 2

UPCA: The UDWT is used for Step 1 and PCA is used for Step 2

Wav: The DWT is used for Step 1 and a DWT with SURE is used for Step 2

UWav: The UDWT is used for Step 1 and a DWT with SURE is used for Step 2.

1) Wavelet-based denoising: Wav and UWav: The SURE introduced by Donoho and Johnstone [18] is a method to compute the scale-dependent wavelet threshold, which, in turn, is the basis for our denoising, i.e., the building of the matrix $\mathbf{P}$ in (3) and (6). The soft thresholding method described in [27] is used to implement SURE in the Wav, UWav Methods. ${ }^{1}$ The soft thresholding reduces border effects that might be encountered in a hard threshold method.

The biorthogonal B-spline wavelet along with analysis and reconstruction filters of orders of $(N h=4, N g=4)$, and a number of scales of $S c=5$ has been chosen for $\mathcal{W}$ and for $\mathbf{H}$ when using the Methods Wav and UWav. The choice of this specific type of wavelet is driven by our experimental data in Section VII-B and has been shown to be efficient for denoising ERP signals [9].

2) PCA-based denoising: PCA and UPCA: The PCA noise reduction is implemented according to the method described in [14] and [28], in which the signal to be denoised is first mapped into a state space using a nonlinear time series embedding technique [29]. The main advantage of this technique is to spread the noise in an approximately uniform manner surrounding the signal state-space manifold, and thus, making it easier to separate the useful information from the noise. The state-space trajectory obtained from the embedding is then decomposed using a PCA (which makes use of the singular value decomposition). The singular values are arranged along the diagonal of a nonsquare matrix, and are used to weight the different principal components. The selection of a restricted number of these weights, i.e., building the matrix $\mathbf{P}$ in (3) and (6), reduces the fluctuations contained in the other components, and hence, the noise in the signal under analysis. This method requires the selection of the embedding dimension $m$ and reconstruction delay $J$. The embedding dimension $m$ has been estimated using a modified version of the false nearest neighbors [30] yielding an upper estimate dimension of $m=30$,

${ }^{1}$ We used the Matlab wavelet toolbox.

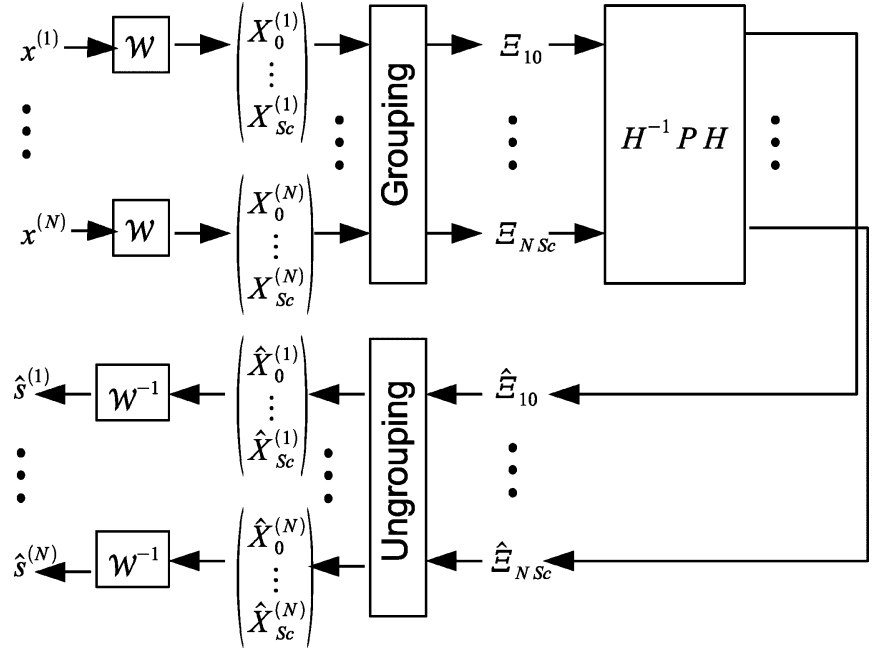

Fig. 3. Graphical representation of the flow of processing. The grouping box refers to the grouping of the wavelet coefficients $\Xi_{i j}(k)$, as shown in Fig. 2.

which was thus subsequently fixed for the PCA, UPCA Methods. ${ }^{2}$ The delay $J$ is chosen as the abscissa of the signal autocovariance function when it drops by $1 / e$ of its maximal value [29]. The PCA in PCA and UPCA Methods uses a predetermined projection dimension of 2. The reason for this choice is that the wavelet coefficients $\boldsymbol{\Xi}_{i j}$ are rather slow varying, and thus, a low dimensional projection space is appropriate for the PCA and UPCA Methods. Optimal selection of the subspace projection dimension requires some deeper analysis of the wavelet coefficient dynamics and is beyond the scope of this paper.

3) Step 3: Inverse DWT or UDWT: Once we have obtained the noise reduced wavelet coefficients $\hat{\boldsymbol{\Xi}}_{i j}$, we can regroup them according to each event $k$ for each scale, and the coefficients $\hat{X}_{j}^{(k)}(i)=\hat{\Xi}_{i j}(k)$ can now be used to build the vector

$$
\left(\hat{\overline{\mathbf{X}}}^{(k)}\right)^{T}=\left(\left(\hat{\mathbf{X}}_{0}^{(k)}\right)^{T}, \ldots,\left(\hat{\mathbf{X}}_{S c}^{(k)}\right)^{T}\right) .
$$

The inverse wavelet transform is finally applied to each individual event's set of wavelet coefficients $\hat{\overline{\mathbf{X}}}^{(k)}$, as given in (5)

$$
\hat{\mathbf{S}}^{(k)}=\mathcal{W}^{-1} \hat{\overline{\mathbf{X}}}^{(k)}
$$

where $\mathcal{W}^{-1}$ represents the (left pseudo)-inverse wavelet matrix, and $\hat{\mathbf{S}}^{(k)}$ the $k$ th noise-reduced event. Fig. 3 summarizes our three-step approach. We present two additional classical denoising methods in the next section for comparison purposes.

\section{Two Other Classical Denoising TechniQues}

To compare our approach to conventional event-by-event noise reduction methods, we have performed wavelet SURE and PCA-based denoising on the events $\mathbf{x}^{(k)}$, i.e., on each time series (TS). For this reason, we use the suffix TS to differentiate

\footnotetext{
${ }^{2}$ This specific choice is linked to our data and must be adapted based on user's data.
} 
these methods from the previously described ones. In this case, the denoising can be mathematically expressed as

$$
\hat{\mathbf{s}}^{(k)}=\left(\mathbf{H}^{-1} \mathbf{P} \mathbf{H}\right) \mathbf{x}^{(k)}, \quad \text { for } \quad k=1, \ldots, N
$$

which corresponds to the following two additional Methods depending on $\mathbf{H}$

PCATS : when $\mathbf{H}$ is a PCA transform. We have used a minimum description length (MDL) criterion to select the relevant principal component (see after for a description of the method) and build the corresponding projection matrix $\mathbf{P}$,

WavTS : when $\mathbf{H}$ is a wavelet transform. We have again used the SURE method to shrink the wavelet coefficients (see after for a description of the method).

The PCA in the PCATS Method uses the same procedure as in the PCA and the UPCA ones, but the MDL criterion [31], [32] was used for selecting the best subspace projection dimension. The MDL criterion enables to select the subspace containing most of the information, and is based on the use of the singular values of the trajectory matrix formed by $\mathbf{x}^{(k)}$, or equivalently, by the eigenvalues of the autocovariance matrix of $\mathbf{x}^{(k)}$ [14], [28].

\section{Performance Measure}

The noise reduction methods have to be assessed in an objective way. In situations where the clean signals $s^{(k)}(n)$ of trial $k$ can be accessed, the normalized mean square error (NMSE) is used to measure the denoising performance with $\epsilon^{(k)}(n)=s^{(k)}(n)-\hat{s}^{(k)}(n)$, as given Eq. (7)

$$
\operatorname{NMSE}_{\text {Method }}(s, \hat{s})=\frac{E\left[\left\langle\left(\epsilon^{(k)}(n)\right)^{2}\right\rangle\right]}{\left.E\left[\left\langle\left(s^{(k)}\right)^{2}(n)\right\rangle\right]+E\left[\left\langle\left(\hat{s}^{(k)}\right)^{2}(n)\right\rangle\right)\right]}
$$

which is bounded between 0 and $1,\langle$.$\rangle is the time averaging$ operation and $E[$.$] the statistical averaging across the k$ trials. The NMSE gain is defined as

$$
\Delta \operatorname{NMSE}_{\text {Method }}(s, \hat{s})=\operatorname{NMSE}(s, x)-\operatorname{NMSE}_{\text {Method }}(s, \hat{s})
$$

where the subscript Method refers to one of the six methods described in Section III, and $x$ is the noisy signal. The NMSE gain is a measure of the reduction of noise achieved by the method under consideration: the larger the $\triangle \mathrm{NMSE}_{\mathrm{Method}}$, the better the performance.

\section{DATA CREATION}

\section{A. Synthetic Signals}

To validate our method, $N=100$ simulated ERPs are used. The synthetic ERP, $s_{\mathrm{Syn}}^{(k)}(n)$, for event number $k=1, \ldots, N$ and time index $n=1, \ldots, L=128$, is constructed as a sum of Gaussian signals to represent the main ERP waves: P1, N2 and, P3 [9]

$$
\begin{aligned}
s_{\mathrm{Syn}}^{(k)}(n) & =\operatorname{ERP}^{(k)}(n)+\eta^{(k)}(n) \\
\operatorname{ERP}^{(k)}(n) & =g_{1}^{(k)}(n)-g_{2}^{(k)}(n)+g_{3}^{(k)}(n)
\end{aligned}
$$

where P1 corresponds to $g_{1}^{(k)}(n), \mathrm{N} 2$ corresponds to $-g_{2}^{(k)}(n)$, and $\mathrm{P} 3$ corresponds to $g_{3}^{(k)}(n)$, with

$$
g_{i}^{(k)}(n)=\exp \frac{-\left(n-t_{i}(k)\right)^{2}}{F_{s}^{2} \sigma_{i}^{2}}, \quad \text { for } \quad i=1, \ldots, 3 .
$$

The standard deviations of the Gaussian signals $g_{i}^{(k)}(n)$ have been fixed to $\sigma_{1}=35 \mathrm{~ms}, \sigma_{2}=35 \mathrm{~ms}$, and $\sigma_{3}=106 \mathrm{~ms}$ in order to mimic a real visual evoked potential, and the sampling frequency to $F_{s}=128 \mathrm{~Hz}$. The $g_{i}^{(k)}(n)$ peak time locations $t_{i}(k)$ fluctuate from event to event in the following intervals: $I_{1}=[90,125] \mathrm{ms}, I_{2}=[126,155] \mathrm{ms}$, and $I_{3}=[400,650] \mathrm{ms}$ to simulate the event-by-event latency jittering effect found in ERP studies. We have simulated three different latency jittering effects, as shown later. The laws (12) (Sine), (13) (Pulse), and (14) (Sigm) are used to model three different nonstationary aspects across the multievents: for each event $k$, there exists a position of the three wave peaks $t_{1}(k), t_{2}(k), t_{3}(k)$, according to

$$
\begin{aligned}
& t_{i}(k)=D_{i}+A_{i} \sin (\omega k) \\
& t_{i}(k)=D_{i}+A_{i} \exp \frac{-(k-N / 2)^{2}}{\left(2 \kappa^{2}\right)} \\
& t_{i}(k)=D_{i}+A_{i}\left(1-\exp \left(-\lambda\left(k-\frac{N}{2}\right)\right)\right)^{-1}
\end{aligned}
$$

with $D_{i} \in I_{i}$ arbitrarily chosen and $A_{i}=\bar{I}_{i} / 3\left(\bar{I}_{i}\right.$ is the middle point of $\left.I_{i}\right)$. Each $D_{i}$, for $i=1,2,3$, is the offset of the peak-time location of the waves $\mathrm{P} 1, \mathrm{~N} 2$, and $\mathrm{P} 3$, and $A_{i}$ 's are the amplitudes of the corresponding event-by-event fluctuations. These laws are exemplified in Figs. 5(b) and 6(b) for the Puls (13) and Sine (12), where we can see the fluctuations of the different waves from event to event.

The background noise $\eta^{(k)}(n)$ is added to the ERP signal $\operatorname{ERP}^{(k)}(n)$ and is constructed using a surrogate method [33] from real EEG signals extracted during prestimulus, artefact free, time periods. The so-constructed noise has a highly colorful content (not white noise) due to its intrinsic EEG nature, yielding more realistic noise reduction performance figures. In this quasi-ideal situation (but more realistic than the classical Gaussian white noise), the random background noise is neurophysiologically meaningless (because of the phase randomization in the surrogate data computation) except that its frequency spectrum is similar to a real set of measurements, and thus, it is expected that the results from these simulations are upperbounded.

\section{B. Human Event-Related Potentials}

One of the most widely used tasks to study cognitive processes with ERPs is the oddball task. In a typical visual oddball task, a series of stimuli are presented on a computer monitor with the probability of occurrence commonly between $10 \%$ and $20 \%$ among a collection of foils. A discriminating key press or simply counting can be used to ensure attendance to the task. The diversity of the foiled stimuli may be limited to a single alternative, i.e., $\mathrm{X}$ and $\mathrm{O}$ are presented with $\mathrm{X}$ as the oddball, or a few different foils may be used, i.e., the letters S, V, and P. 
Fourteen female participants were recruited for this experiment, and they received an incentive of course credit. The mean age was 21.0 years old with the standard deviation of 4.0 years (range 18-31 years old). None of the participants reported history of neurological or mental disorder. All participants read and signed a consent form that described the basic procedures of the experiment.

The stimuli consisted of the following letters X, G, T, and A in times new roman font. The letter size is printed at an angle of $0.8^{\circ}$ in height and width by using the Presentation software (Version 9.90, www.neurobs.com). A $2300 \mathrm{MHz}$ advanced micro devices (AMD) computer was used with a stimulus onset error of less than $1 \mathrm{~ms}$. The monitor was encased in a wire mesh, and the room was shielded (Faraday cage) with both being earth-grounded. All main power and recording equipments were outside the room. A button pad with left and right buttons was placed on the participants' laps.

The participants arrived and signed the consent form and prepared for the EEG recording. They were seated in a padded lounge chair with the distance of $1 \mathrm{~m}$ from the computer monitor. The letter $\mathrm{X}$ was designated as the oddball stimulus with the remaining as foils. The oddballs were presented for $15 \%$ of the total trials of 265 , i.e., $L \approx 40 \mathrm{Xs}$ were presented. The foils were randomly picked and presented in $85 \%$ of the trials. Each stimulus was presented for $1500 \mathrm{~ms}$ followed by a $1000 \mathrm{~ms}$ blank screen. Half of the participants were instructed to press the left button on the pad if an X was presented and the right button for the other letters, the other half of the participants had their keys reversed. They were also shown their free running EEG just prior to the task and the effects of moving, blinking and eye movements to show the importance of sitting still and trying to blink only when the blank screen was present. A short rest was given for every 100 trials.

A Neuroscan Synamps-2 system was used for the EEG data acquisition. Data were continuously recorded, and later, epoched in the range of $100 \mathrm{~ms}$ prestimulus to $1000 \mathrm{~ms}$ poststimulus. A 32-channel cap was used with $\mathrm{Ag} / \mathrm{AgCl}$ contacts and eye electrodes. The electrodes were placed above and below the right eye to record vertical eye movements and blinks. Two electrodes were placed at the outer canthi of each eye to record horizontal eye movements. For the cap, an electrogel was placed in each electrode cup to form a connection bridge to the participant's scalp. All electrode impedances were brought to below $5 \mathrm{k} \Omega$ prior to recording and were checked immediately after recording. The cutoff frequency of the bandpass filter was in the range of $0.15-100 \mathrm{~Hz}$, and that of a notch filter was $50 \mathrm{~Hz}$. Data were digitized at $1000 \mathrm{~Hz}$ and resampled at $F_{s}=200 \mathrm{~Hz}$ using 16-bit resolution. The epoched files were saved to a disk without performing other form of artifact reduction, i.e., blink attenuation, prior to digitally processing the signals.

\section{RESULTS}

\section{A. Synthetic Signals}

Fig. 4 shows the average of the NMSE gain for different Methods across 50 Monte-Carlo simulations with the SNR ranging from -5 to $20 \mathrm{~dB}$ using the three different latency jittering in

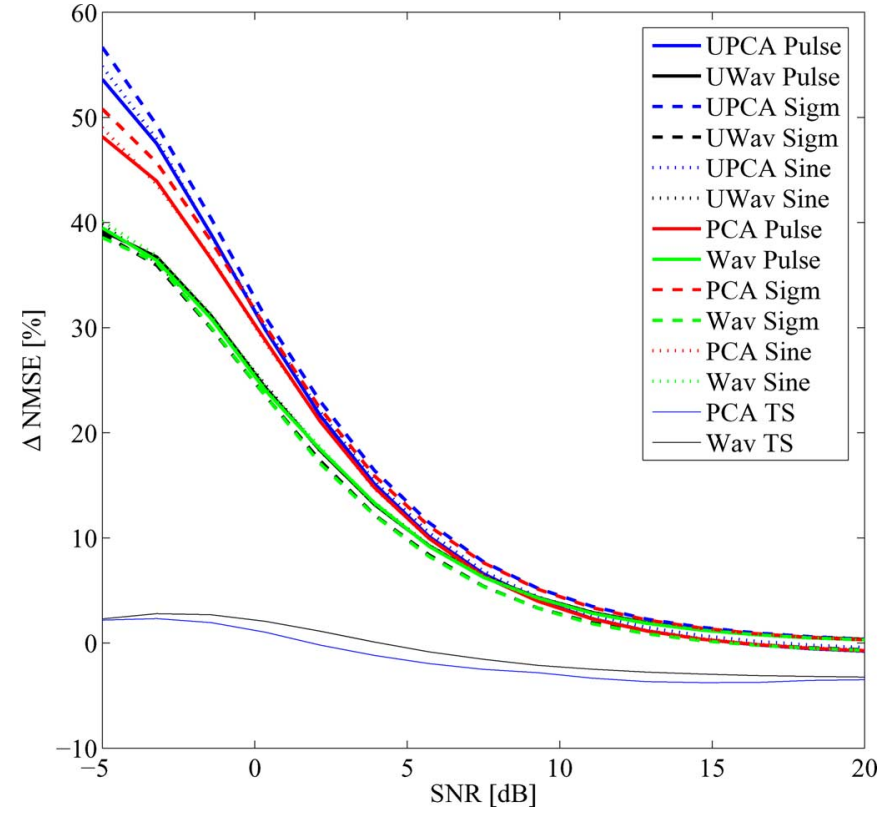

Fig. 4. $\triangle$ NMSE $_{\text {Method }}$ for all the Methods for an SNR varying from -5 to $20 \mathrm{~dB}$ and for the three laws (12), (13), (14), which are referred to as Sine, Pulse, and Sigm. We have fixed the law parameters as: $\kappa=\sqrt{25}, \omega=2 \pi / N$, and $\lambda=4 / N$. Fifty Monte-Carlo simulations have been used and the average results are presented. The methods PCATS and WavTS give results on each signal (12), (13), (14), which are indistinguishable.

(12), (13), and (14). The SNR is defined as ten times the base-10 logarithm of ratio of the variance of the signal $\operatorname{ERP}^{(k)}(n)$ to the variance of the noise $\eta^{(k)}(n)$.

1) Multievent Denoising: It can be observed in Fig. 4 that there is an appreciable difference between the UPCA and PCA Methods, i.e., the UDWT performs better than the DWT because $\triangle \mathrm{NMSE}_{\mathrm{UPCA}} \geq \Delta \mathrm{NMSE}_{\mathrm{PCA}}$. However, this difference quickly drops as the SNR becomes larger than $0 \mathrm{~dB}$. This result was predictable, as explained in Section II. However, what is more surprising is the good performance of the DWT despite its time-shift noninvariance, which actually makes it very effective for fast denoising of multievents. For the experimental results, we will thus concentrate on the Wav and PCA Methods, which use the DWT in Step 1. This conclusion is further confirmed by the visual inspection of Figs. 5 and 5(c)-(f) for the (U)Wav and the (U)PCA Methods where the slight improvement using UDWT is largely compensated by the processing speed of the DWT.

Fig. 4 indicates that there is no significant difference between the UWav and Wav Methods at any SNR level. They always perform worse than the UPCA or PCA Methods. Even the Pulse jittering shows better results with the PCA-based denoising, while we could have expected the wavelet to be more suitable for this type of brief jittering effect (at least, when using the UDWT in Step 1).

Figs. 5(a)-(h) and 6(a)-(h) show the multievent signals on a set of gray scale images where the $Y$-axis represents the event number and the $X$-axis the time. The gray scale is proportional to the signal's amplitude, i.e., large negative values are darker than large positive values. 

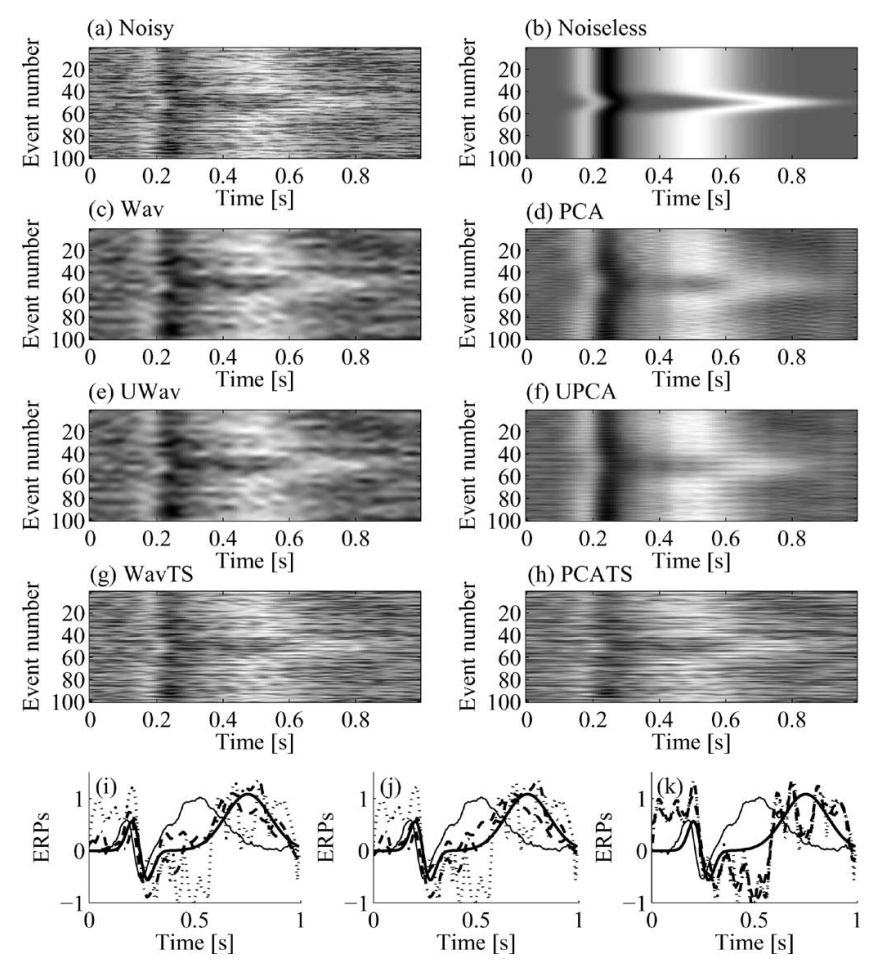

Fig. 5. Multievent signals for the Pulse law (13) with $\kappa=\sqrt{25}$. (a) Noisy signals ( $N=100, \mathrm{SNR}=-3 d B$. (b) Noiseless signals. (c)-(d) Noise reduced using the Wav and PCA Methods. (e)-(f) Noise reduced using the UWav and UPCA Methods. (g)-(h) Noise reduced using the WavTS and PCATS Methods. (i) PCA (--), Wav (-.); (j) UPCA (- -), UWav (-.); (k) PCATS (- -), WavTS (-.). (i)-(k) Display time signals for the trial $i=N / 2$ together with the noisy signal (dotted bold line), the average signal (continuous thin line).

Figs. 5 and 6(c) and (d) show the denoising results using the Wav and PCA Methods. Figs. 5 and 6(e) and (f) show the results of the denoising using UWav and UPCA. Fig. 6 has been produced with an $\mathrm{SNR}=-3 \mathrm{~dB}$, while Fig. 6 with $\mathrm{SNR}=3 \mathrm{~dB}$. Figs. 5 and 6(c)-(f) show that the (U)Wav and (U)PCA Methods perform quite well with smoother results for the (U)PCA Methods. The (U)PCA Methods tend to have a better localization in the time, while (U)Wav Methods are better at localizing the event number $k$. For each event, the (U)PCA Methods provide a smoother denoised signal: a signal with a more compact frequency content. In Fig. 6(i)-(j), the (U)PCA and (U)Wav results are almost indistinguishable from the true signal $\operatorname{ERP}^{(k)}(n)$.

Neuroclinicians are used to average ERPs to reduce the noise in these signals. The effect of averaging is indeed to smooth the signals, but at the expense of information distortion in the single event due to their nonstationarity. This is exemplified in Figs. 5 and 6(i)-(k) where the $N$ events average (fine continuous line) is displayed against single noiseless event (bold line) and noise reduced signals. These results show that the averaging technique distorts the information content in single event and that the multievent denoising must be preferred.

The experimental results in the next section show that while the quantitative results using the NMSE gain favor the PCA approach, the Wav Method might have some advantages when visual inspection of these multievent is used. A detailed clinical analysis of these methods is beyond the scope of this methodological paper.
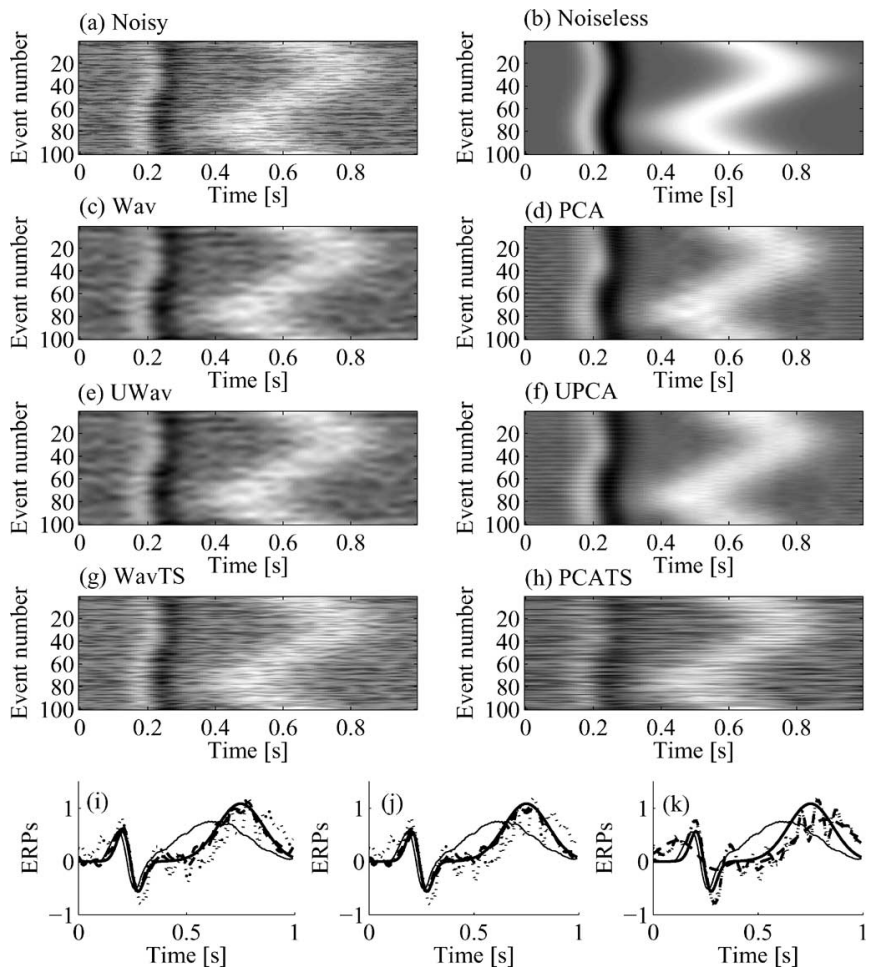

Fig. 6. Multievent signals for the Sine law (12) with $\omega=2 \pi / N$. (a) Noisy signals $(N=100, \mathrm{SNR}=3 d B$. (b) Noiseless signals. (c)-(d) Noise reduced using the Wav and PCA Methods. (e)-(f) Noise reduced using the UWav and UPCA Methods. (g)-(h) Noise reduced using the WavTS and PCATS Methods. (i) PCA (- -), Wav (-.); (j) UPCA (- -), UWav (-.); (k) PCATS (- -), WavTS (-.). (i)-(k) Display time signals for the trial $i=N / 4$ together with the noisy signal (dotted bold line), the average signal (continuous thin line).

2) Event-by-event vs multievent denoising: The PCATS and WavTS Methods show the lowest NMSE gain, as expected, based on: 1) the fact that the full knowledge of all the events and their event-by-event fluctuations are not used in these methods and 2) the realistic noise signal that we have used is quite spectrally colored, thus either the PCATS or WavTS Method considers these rhythms as part of the signals' information. The PCA or Wav Method performs the denoising on the set of wavelet coefficients $\boldsymbol{\Xi}_{i j}$, which might contain correlation, but on the set of $N$ wavelet coefficients and not in the time domain. In other words, the fact of using a DWT on several events embeds the set of events in a space for which the time correlations found in each individual events are lost at the benefits of compactness of the information in the set of $N$ multievent wavelet coefficients.

Figs. 5(g) and (h) and 6(g) and (h) show the results of the denoising using WavTS and PCATS, respectively. Both the images clearly show that they cannot attenuate the noise as in the case of (U)Wav and (U)PCA Methods. This is further shown in the time series of the denoised events in Figs. 5(k) and Fig. 6(k). In Fig. 6(k), we can observe that the PCATS and WavTS cannot distinguish the background color noise activity from the true single event.

\section{B. Human ERPs}

The ERP signals have a typical SNR below $0 \mathrm{~dB}$ that make them very difficult to analyze and fully justify noise reduction 
(a)

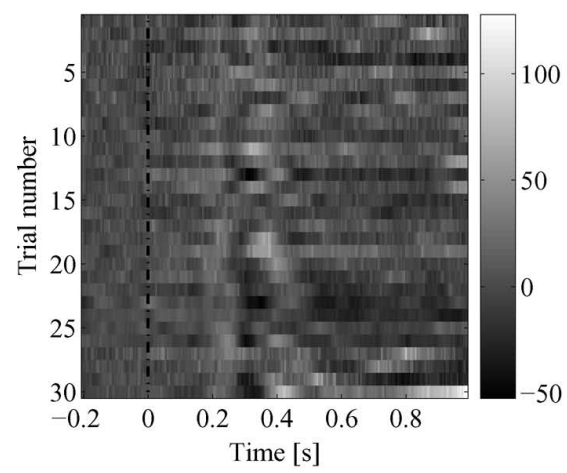

(c)

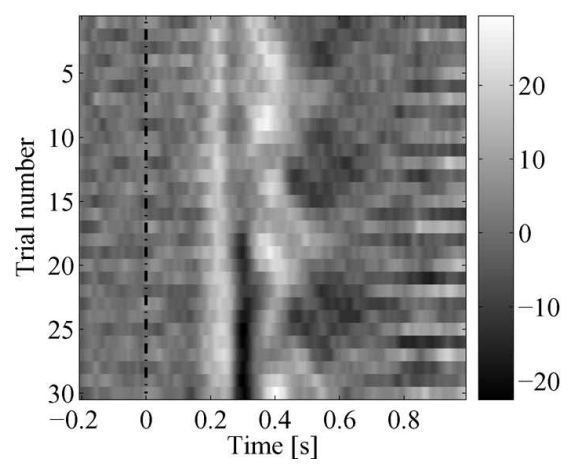

(b)

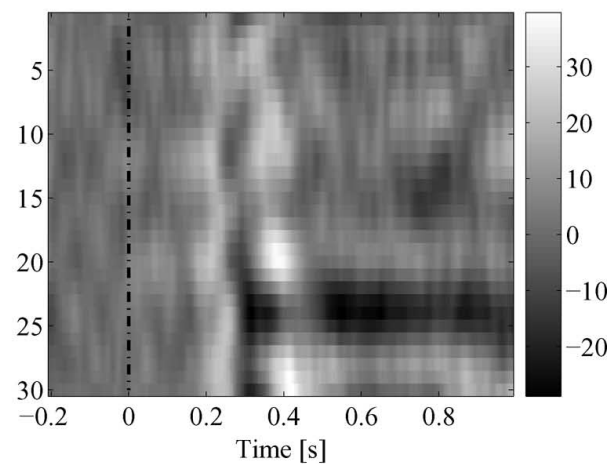

(d)

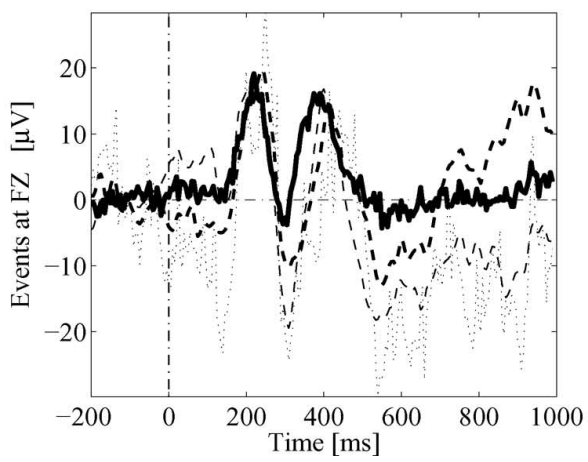

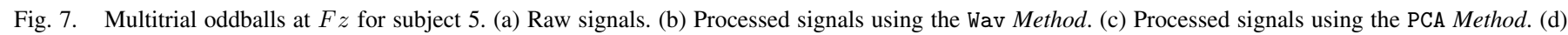

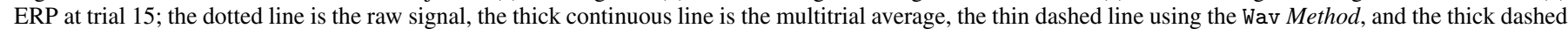
line using the PCA Method.

(a)

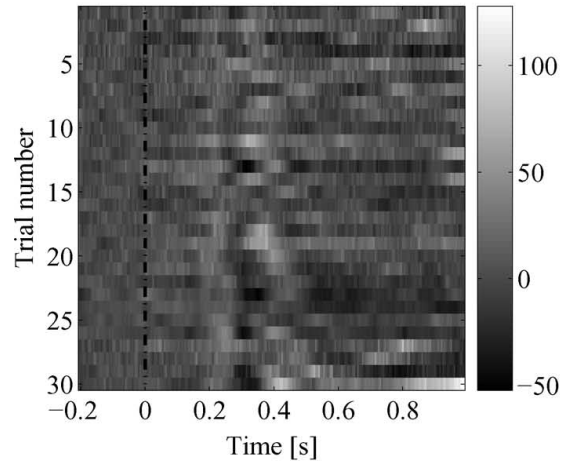

(c)

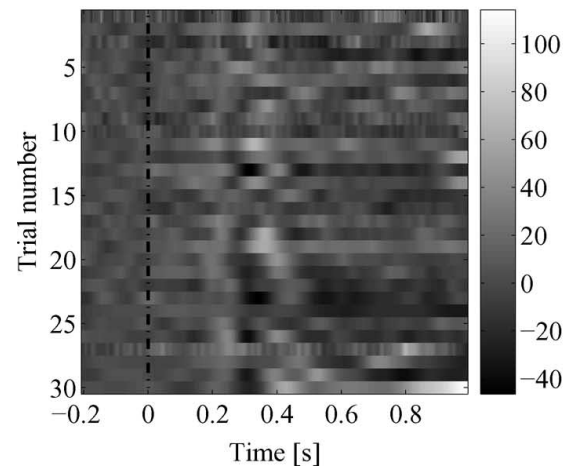

(b)

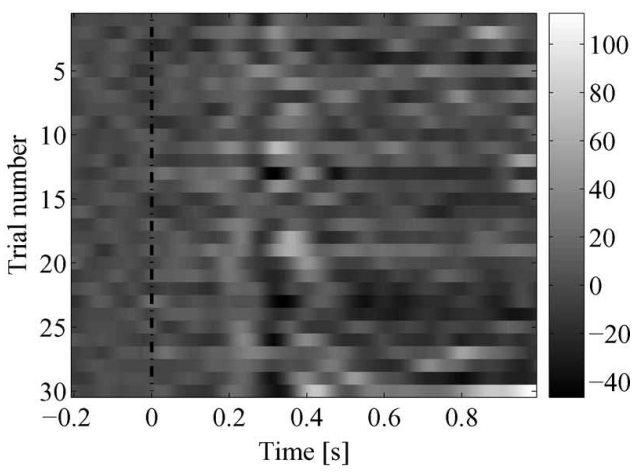

(d)

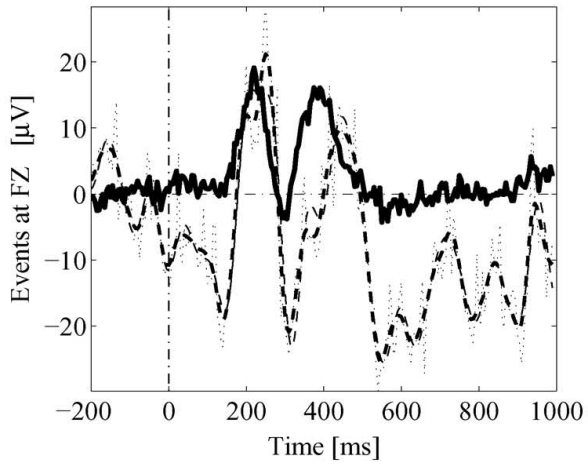

Fig. 8. Multitrial oddballs at $F z$ for subject 5. (a) Raw signals. (b) Processed signals using the WavTS Method. (c) Processed signals using the PCATS Method.

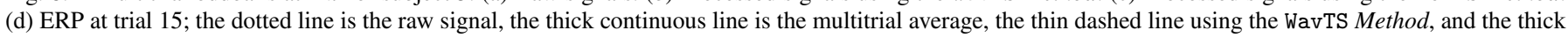
dashed line using the PCATS Method. The color bar indicates the amplitude in microvolts. 

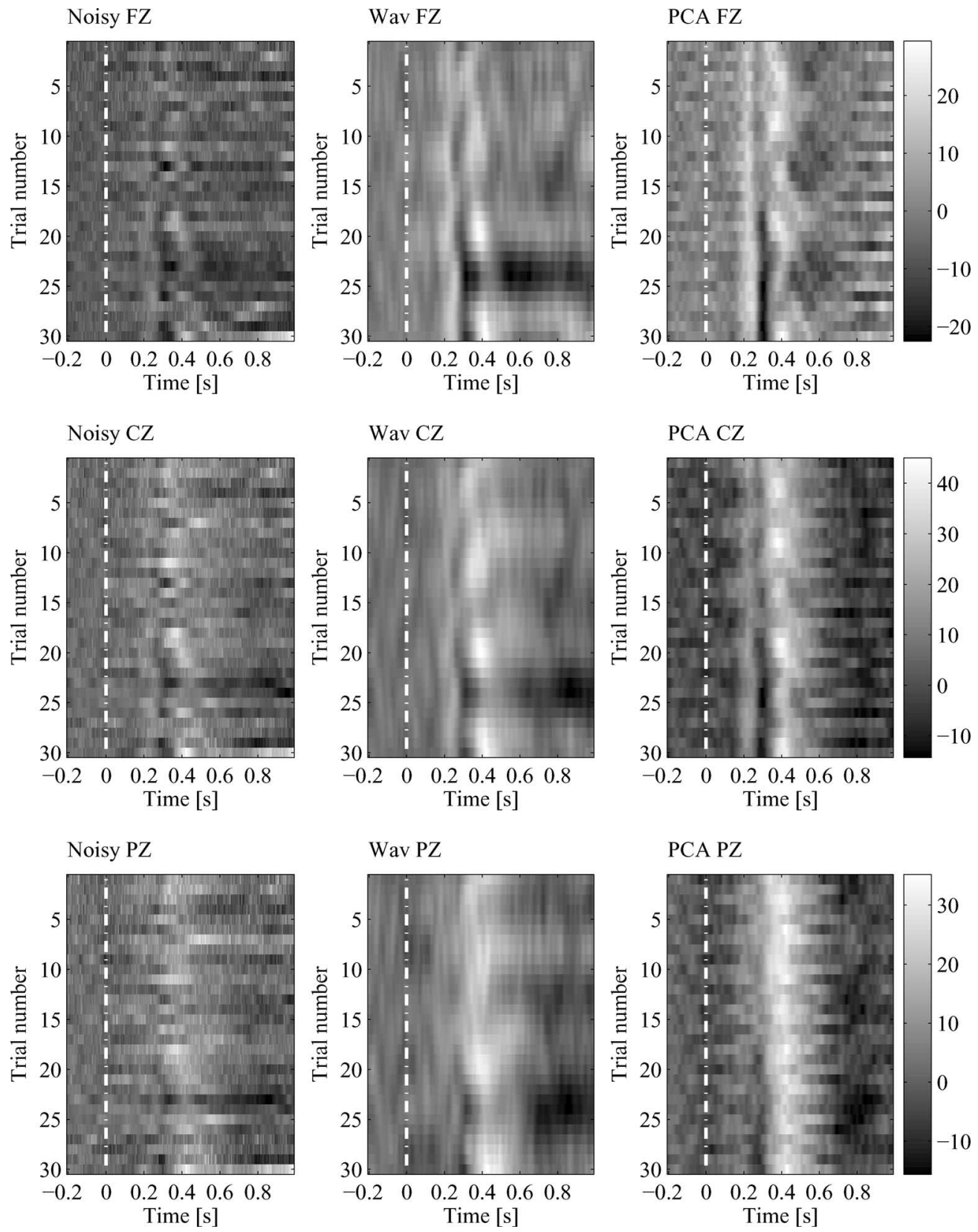

Fig. 9. Multitrial oddballs at $F z, C z$, and $F z$ for subject 5. All the images display the trial-by-trial matrix on a gray scale image. The first line are the trial at $F z$, the second line at $C z$, and the third at $P z$. The first column are the raw signals, the second are the denoised trials with the Wav Method, and the last are the denoised trials with the PCA Method. The color bar indicates the amplitude in microvolts.

techniques such as the one proposed in this paper. The P3 is typically shown at midline electrodes (as it rarely shows lateralization), and $F z, C z$, and $P z$ are the standard sites compared over most studies of this ERP component [34]. Applying our different denoising methods to the ERP will reveal the advantages of the new strategy. As explained in the previous section, we present only the results using the DWT in Step1 for the multievent denoising, i.e., the Wav and PCA Methods. We also present the results of the WavTS and the PCATS Methods for visual comparison purposes on one electrode site $F z$.

Repetition in a simple oddball task can engage frontal processes (P3a effect) in the automatic allocation of attention [35]. The usual P3 oddball effect would, thus, be expected to shift from the parietal region to more central sites (that is $C z$ and $F z)$ as this task proceeds. We examine $F z$ in particular for one subject to illustrate this possible effect. Two other examples are also illustrated later showing similar effects with details provided at all three midline electrodes.

The stimulus takes place at $t=0$ as indicated by the vertical dotted line. The ERPs have been baseline corrected using the 200 ms signal preceding the stimulus. Figs. 7 and 8 show the processed oddball multitrials ERP for one subject at the electrode position $F z$. The PCA and Wav Methods have been used for Fig. 7, and the PCATS and WavTS Methods for Fig. 8. A very distinctive improvement is seen when observing Fig. 7(b) and (c), where two ridges are perfectly visible, as opposed to the 

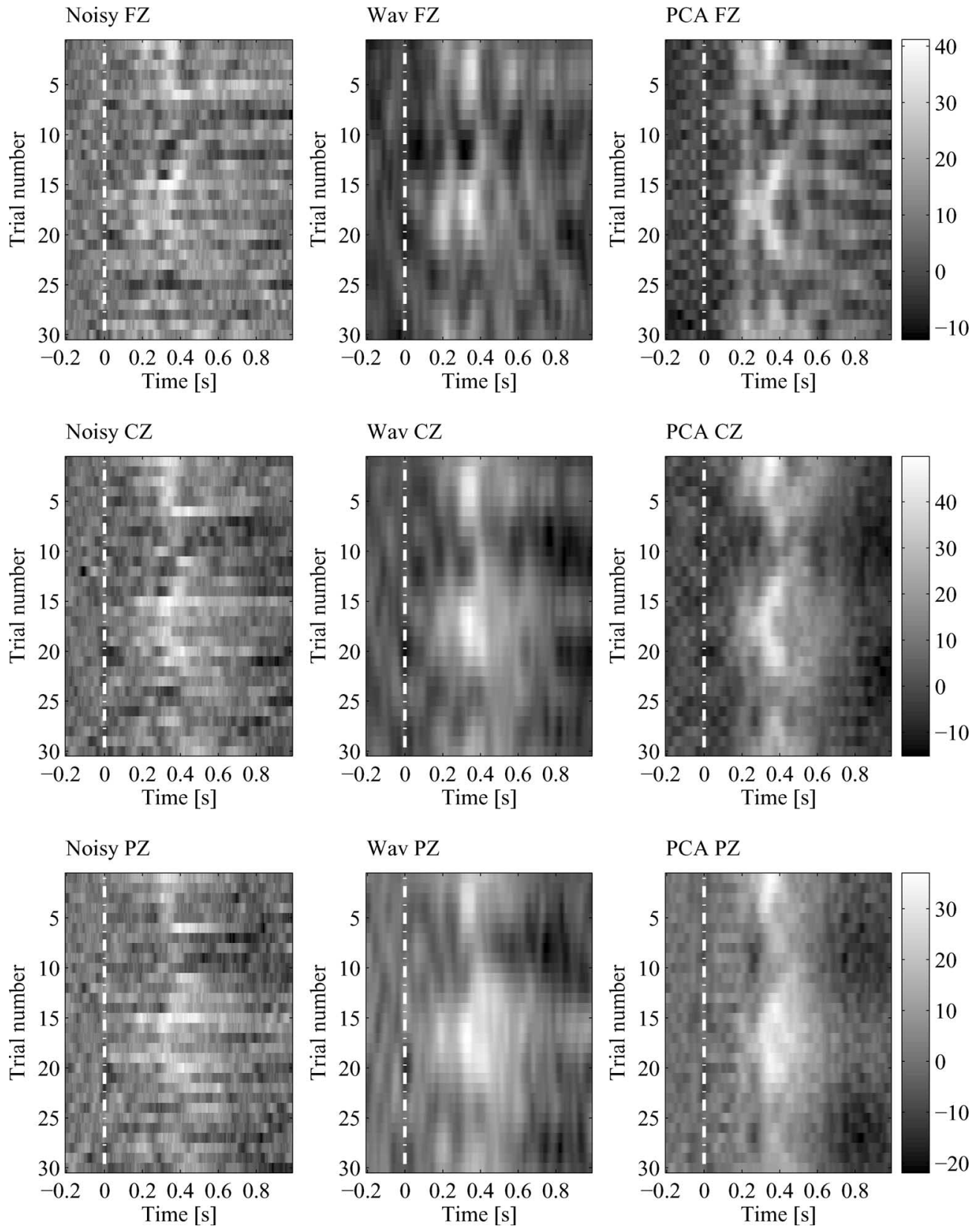

Fig. 10. Same as Fig. 9 for subject 3 .

original ERPs in Fig. 7(a). As seen in Fig. 8, neither the PCATS nor WavTS Methods can provide quantitative improvement as compared with the Wav or PCA Methods.

While the PCA and Wav Methods yield different visual results, they are able to show distinctive trial-by-trial time-shift modulation of the ERP wave complexes. From Fig. 7(b) and (c), at about $-200 \mathrm{~ms}$, the signals are less structured in the time domain, i.e., broader frequency spectrum, as compared with the poststimulus. Fig. 7(b) and (c) shows two crests (from top to bottom) and illustrates changes in the morphology of the ERP to the oddball events. The role of the frontal cortex is to execute plans and actions to monitor and refine cognitive activities. Attentional allocation and control are one of these executive functions [36]. It can also be noted that the distance between the $\mathrm{P} 2$ and $\mathrm{P} 3$ peaks fluctuates with the trial number that fur- ther broadens the waveform. The reason for this modulation effect is that the oddball trials are randomly distributed over the duration of the recording, and they only account for about $15 \%$ of the total 265 trials, while their appearance during the recording session is chronologically presented. This modulation effect can be caused by the memory effects of the oddball events.

This effect, even though not as clear in all the trial subjects, is strongly present in about $53 \%$ of the 15 subjects. Figs. 9 to 11 display the multitrial set at the electrode positions $F z, C z$, and $P z$ for three subjects using the Wav and PCA Methods. In these figures, we can appreciate the different characteristics of the Wav and PCA Methods: the Wav Method gives a smoother image than the PCA Method. This gives a feeling of flow from event to event when looking at the Wav Method results, while this can 

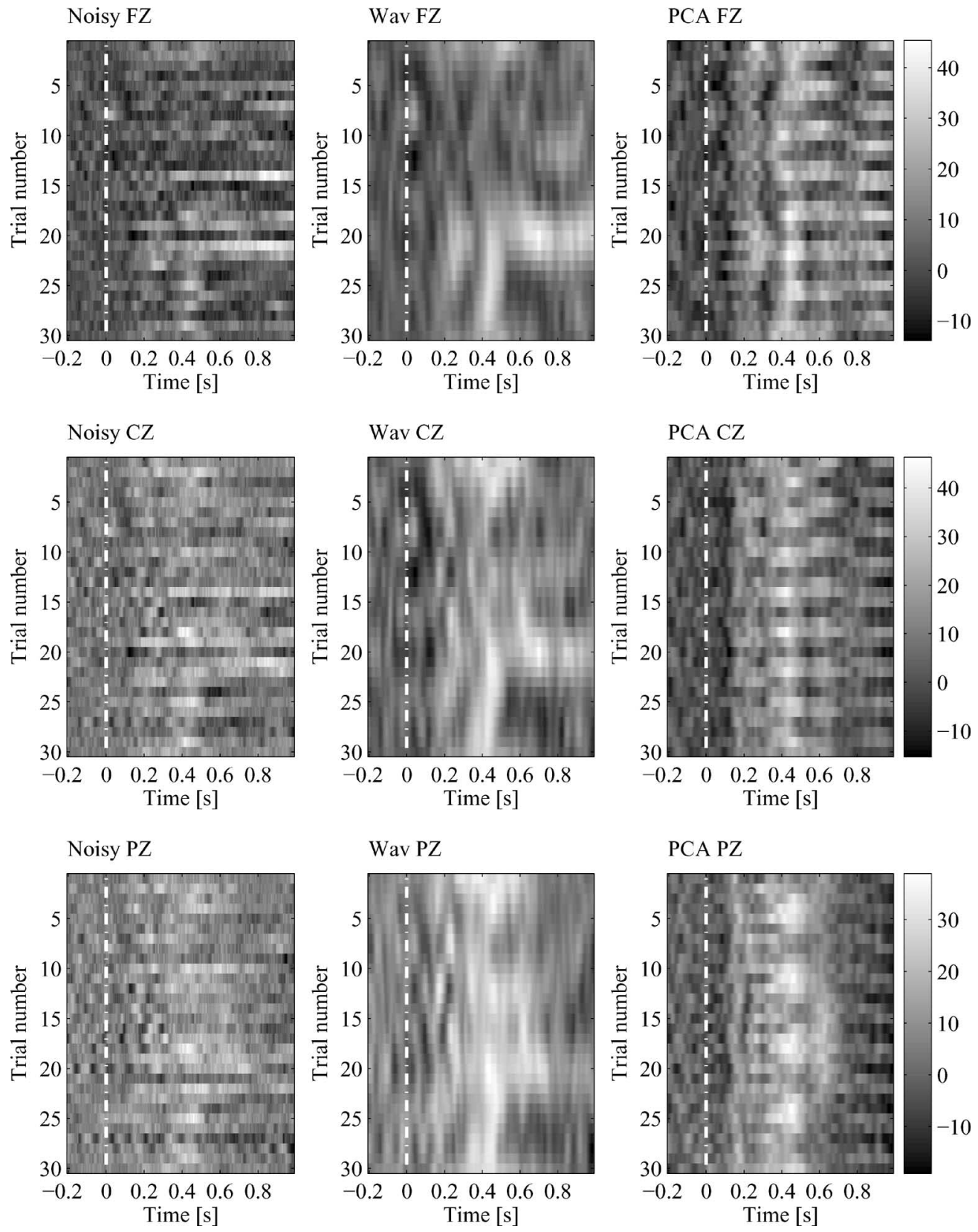

Fig. 11. Same as Fig. 9 for subject 1.

be an artifact of the method. The PCA Method does not present such a flow, but tends to increase alternate patterns of high and low activity (dark and light regions) across events.

The denoising methods reveal interesting features hidden in the raw noisy signals, as described earlier, especially on the frontal and central regions. These figures show the emergence (and fading) of ERP patterns that suggest nonstationary cognitive processes in this simple detection task. The P3a in central and frontal sites (for all three subjects illustrated) becomes more distinguished from a P2 component. One possibility is that this may reflect these subjects waxing and waning of attention (we have observed in previous use of this task that subjects show a fluctuation in attentiveness-reflecting a monotonous task carried out in a dimly lit room). The oddball task performed in this study required discrimination between four letters $\mathrm{X}, \mathrm{G}$, $\mathrm{T}$, and $\mathrm{A}$, with the letter $\mathrm{X}$ being the rare target event. With repeated trials, there appears to be changes in the frontal separation and amplitude of the N1-P2 and N2-P3 complexes. The N1-P2 complex has been related to attentional processes [37], possibly shifting to a specific feature of the target that promotes easy discrimination. With practice, this sharpens in the crest after about half of the trials. The magnitude of the N2-P3 complex has been related to decision certainty and has shown a shift from parietal to more frontal regions with task automation [38], [39]. The P2 itself has been scarcely studied, and the present results, showing significant improvement in the SNR for this component at frontal locations, open new directions for researchers to uncover the source and function of this ERP. 
Visual inspection of Figs. 9-11 would suggest a natural preference to the Wav Method against the PCA Method due to its qualitative smoothness across the multitrials, while the situation is quite the opposite when we look at the signals in the time domain trial by trial, as in Fig. 7(d), for instance. Also, when comparing Figs. 5(c) and (d) and 7(b) and (c), it seems that the PCA and the Wav gives different smoothing results, but this is only due to the small number of events used in 7(b) and (c). The conclusion is that while the NMSE gives a quantitative advantage for the PCA Method, we obviously need an other measure when assessing Figs. like 9-11. This new measure must be somehow complementary to the NMSE.

\section{CONCLUSION}

A new noise reduction algorithm has been presented for use on multitrial or sets of patterns extracted from rhythmical signals, called a multievent signal. This method does not involved any manual intervention during the denoising process, while requiring the predetermination of few parameters, and can thus be considered as semiautomatic. This method involves two subspace decompositions: the first one being a wavelet transform on each event, the second one being the basis to perform the actual noise reduction across events' wavelet coefficients and can be implemented using a PCA or a wavelet transform. The decimated and undecimated DWTs are used in the first step. The undecimated DWT performs better than the decimated discrete wavelet at the cost of increased algorithm complexity.

It should be noted that another redundant wavelet transform of interest is the continuous wavelet transform that has been implemented using fast algorithms such as the one presented in [40]. We, thus, recommend the decimated DWT when fast processing of multievent is required, while the undecimated discrete wavelet or continuous wavelet transform would be preferred for higher accuracy.

The choice between PCA or wavelet denoising at the second step depends on the signal under study, but we recommend the PCA whenever the wavelet coefficients across all events have regular patterns. Wavelet denoising, however, shows smoother results when visually assessed on real data with a small number of events.

Using the classical NMSE measure, it has been shown that the new algorithm performs much better than the event-by-event denoising schemes such as PCA-based and wavelet SURE techniques, especially for low SNR. It has also been shown that the new algorithm can reveal the time-varying nature of the events' features. While the NMSE is a good performance measure for a class of signals and features, its limitations has been shown when studying multitrial ERP signals. The main reason for this is that the NMSE has no smoothness measure, while visual inspection tends to promote smooth results. Other performance measures must then be developed for the analysis of multievent signals. An attempt to do so is the S-index that actually measures a state-space synchronization between the denoised signal and a reference signal [44]. Other performance measures based on entropies such as the transinformation (information transfer) between the denoised signal and the noisy signal, or based on ex- pert pattern recognition/classification systems (which can also be based on transinformation criterion) can also be investigated.

Applications on ERP have further revealed a progressive change in the morphology of the N1-P2 [45] and N2-P3 waves at the central frontal sites, as the oddballs are collected. Further research on these data is underway to analyze more thoroughly the effects of attentiveness and memory on the ERP wave patterns, time and scale distributions.

\section{ACKNOWLEDGMENT}

The authors would like to thank the reviewers for their fruitful comments that improved the quality of this paper.

\section{REFERENCES}

[1] D. D. B. D. Rubin, G. Baselli, G. F. Inbar, and S. Cerutti, "An adaptive neuro-fuzzy method (ANFIS) for estimating single-trial movement-related potentials," Biol. Cybern., vol. 113, pp. 63-75, 2002.

[2] A. Effern, K. Lehnertz, T. Schreiber, T. Grunwald, P. David, and C. E. Elger, "Nonlinear denoising of transient signals with application to event related potentials," Phys. D, vol. 140, pp. 257-266, 2000.

[3] A. Effern, K. Lehnertz, T. Grunwald, G. Fernández, P. David, and C. E. Elger, "Single trial analysis of event related potentials: Nonlinear denoising with wavelets," Clin. Neurophysiol., vol. 11, pp. 2255-2263, 2000.

[4] D. O. Walter, "A posteriori 'Wiener filtering' of averaged evoked responses," Electroenceph. Clin. Neurophysiol., vol. 27, pp. 61-70, 1969.

[5] D. J. Doyle, "Some comments on the use of Wiener filtering for the estimation of evoked potentials," Electroenceph. Clin. Neurophysiol., vol. 38, pp. 533-534, 1975 .

[6] S. Cerutti, V. Bersani, A. Carrara, and D. Liberati, "Analysis of visual evoked potentials through Wiener filtering applied to a small number of sweeps," J. Biomed. Eng., vol. 9, pp. 3-12, 1987.

[7] E. A. Bartnik, K. Blinowska, and P. J. Durka, "Single evoked potential reconstruction by means of wavelet transform," Biol. Cybern., vol. 67, pp. 175-181, 1992.

[8] S. Makeig, A. Bell, T.-P. Jung, and T. Sejnowski, "Independent component analysis of electroencephalographic data," Adv. Neural Inf. Process. Syst., vol. 1, pp. 79-82, 1997.

[9] R. Q. Quiroga and H. Garcia, "Single-trial event-related potentials with wavelet denoising," Clin. Neurophysiol., vol. 114, pp. 376-390, 2003.

[10] A. Effern, K. Lehnertz, T. Grunwald, G. Fernández, P. David, and C. E. Elger, "Time adaptive denoising of single trial event-related potentials in the wavelet domain," Psychophysiology, vol. 37, pp. 859-865, 2000.

[11] P. A. Karjalainen, J. P. Kaipio, A. S. Koistinen, and M. Vauhkonen, "Subspace regularization method for the single-trial estimation of evoked potentials," IEEE Trans. Biomed. Eng., vol. 46, no. 7, pp. 849-860, Jul. 1999.

[12] I. T. Jolliffe, Ed. Principal Component Analysis. Springer series in statistics. New York: Springer-Verlag, 1986.

[13] A. Aldroubi and M. Unser, Wavelets in Medicine and Biology. Boca Raton, FL: CRC Press, 1996.

[14] P. Celka, R. Vetter, E. Gysels, and T. Hine, "Dealing with randomness in biosignals," in Handbook of Time Series Analysis. Recent Theoretical Developments and Applications, M. Winterhalder, B. Schelter, and J. Timmer, Eds. Berlin, Germany: Wiley-CVH Verlag GmbH, 2006, pp. 101-141.

[15] M. Browne and T. R. Cutmore, "Low-probability event-detection and separation via statistical wavelet thresholding: An application to psychophysiological denoising," Clin. Neurophysiol., vol. 113, pp. 1403-1411, 2002.

[16] P. Celka and E. Gysels, "Smoothly adjustable denoising using a priori knowledge," Signal Process., vol. 86, pp. 2233-2242, 2006.

[17] S. Akkarakaran and P. Vaidyanathan, "Results on principal component filter banks: Colored noise suppression and existence issues," IEEE Trans. Inf. Theory, vol. 47, no. 3, pp. 1003-1020, Mar. 2001.

[18] D. L. Donoho and I. Johnstone, "Adapting to unknown smoothness via wavelet shrinkage," J. Amer. Statist. Assoc., vol. 90, pp. 1200-1224, 1995

[19] J.-P. Antoine, R. Murenzi, P. Vandergheynst, and S. T. Ali, Twodimensional Wavelets and Their Relatives. Cambridge, U.K.: Cambridge Univ. Press, 2004.

[20] S. Mallat, A Wavelet Tour of Signal Processing, New York: Academic, 1999. 
[21] M. Lang, H. Guo, J. E. Odegard, C. S. Burrus, R. O. Wells, and Jr., "Noise reduction using an undecimated discrete wavelet transform," IEEE Signal Process. Lett., vol. 3, no. 1, pp. 10-12, Jan. 1996.

[22] R. R. Coifman and D. L. Donoho, "Translation-invariant de-noising," in Wavelets Statistics, New York: Springer-Verlag, 1995, pp. 125-150.

[23] J.-C. Pesquet, H. Krim, and H. Carfantan, "Time-invariant orthonormal wavelet decomposition," IEEE Trans. Signal Process., vol. 44, no. 8, pp. 1964-1970, Aug. 1996.

[24] A. Chambolle and B. J. Lucier, "Interpreting translation-invariant wavelet shrinkage as a new image smoothing scale space," IEEE Trans. Image Process., vol. 10, no. 7, pp. 993-1000, Jul. 2001.

[25] C. S. Burrus, R. A. Gopinath, and H. Guo, Introduction to Wavelets and Wavelet Transform: A Primer. Upper Saddle River, NJ: Prentice-Hall, 1998.

[26] M. Vetterli and J. Kovacevic, Wavelets and Subband Coding, Signal Processing Series. Upper Saddle River, NJ: Prentice-Hall, 1995.

[27] D. L. Donoho, "De-noising by soft-thresholding," IEEE Trans. Inf. Theory, vol. 41, no. 3, pp. 613-627, May 1995.

[28] R. Vetter, J.-M. Vesin, P. Celka, P. Renevey, and J. Krauss, "Automatic nonlinear noise reduction using local principal component analysis and MDL parameter selection," in Proc. IEEE EMBS 2003, Cancun, Mexico, vol. 1, pp. 491-496.

[29] H. Kantz and T. Schreiber, Nonlinear Time Series Analysis, Nonlinear Science Series 7. Cambridge, U.K.: Cambridge Univ. Press, 1997.

[30] D. R. Fredkin, "Method of false nearest neighbors: A cautionary note," Phys. Rev. E, vol. 51, pp. 2950-2954, 1995.

[31] J. Rissanen, Stochastic Complexity in Statistical Engineering. Singapore: World Scientific, 1989.

[32] J. Rissanen, "MDL denoising," IEEE Trans. Inf. Theory, vol. 46, no. 7, pp. 2537-2543, Nov. 2000.

[33] T. Schreiber and A. Schmitz, "Improved surrogate data for nonlinearity tests," Phys. Rev. Lett., vol. 77, pp. 635-638, 1996.

[34] M. Fabiani, G. Gratton, and M. G. H. Coles, "Event-related brain potentials: Methods, theory and applications," in Handbook of Psychophysiology, 2nd ed. L. G. Tassinary, J. T. Cacioppo, and G. G. Berntson, Eds. London, U.K.: Cambridge Univ. Press, 2004.

[35] U. Volpe, A. Mucci, P. Bucci, E. Merlotti, S. Galderisi, and M. Maj, "The cortical generators of P3a and P3b: A LORETA study," Brain Res. Bulletin, vol. 73, pp. 220-230, 2007

[36] J. Alvarez and E. Emory, "Executive function and the frontal lobes: A meta-analytic review," Neuropsychol. Revi., vol. 16, pp. 17-42, 2006.

[37] A.-C. Lukaszewicz, L. Garcia-Larrea, and F. Mauguière, "Revisiting the oddball paradigm. Non-target vs neutral stimuli and the evaluation of ERP attentional effects?," Neuropsychologia, vol. 30, pp. 723-741, 1992.

[38] M. Lindin, M. Zurrûn, and F. Diaz, "Changes in P300 amplitude during an active standard auditory oddball task," Biol. Psychol., vol. 66, pp. 153$167,2004$.

[39] J. K. Olofsson and J. Polich, "Affective visual event-related potentials: Arousal, repetition, and time-on-task," Biol. Psychol., vol. 75, pp. 101108, 2007.

[40] M. J. Vrhel, C. Lee, and M. Unser, "Fast continuous wavelet transform: A least-square formulation," Signal Process., vol. 57, pp. 103-119, 1997.

[41] M.-O. Hongler, Y. L. de Meneses, A. Beyeler, and J. Jacot, "The resonant retina: Exploiting vibration noise to optimally detect edges in an image," IEEE Trans. Pattern. Analysis Machine Intell., vol. 25, no. 9, pp. 10511062, Sep. 2003.

[42] T. Ditzinger, M. Stadler, D. Strüber, and J. A. S. Kelso, "Noise improves three-dimensinal perception: Stochastic resonance and other impacts of noise to the perception of autostereograms," Phys. Rev. E, vol. 62, pp. 2566-2575, 2000 .

[43] M. Riani and E. Simonotto, "Stochastic resonance in the perceptual interpretation of ambiguous figures: A neural network model," Phys. Rev. Lett., vol. 72, pp. 3120-3123, 1994.

[44] P. Celka and B. Kilner, "Carmeli's s index assesses motion and muscle artefacts reduction in rowers' electrocardiogram," Physiol. Meas., vol. 27, pp. 737-755, 2006.

[45] T. Grunwald, N. Boutros, N. Pezer, J. von Oertzen, G. Fernandez, C. Schaller, and C. E. Elger, "Neuronal substrate of sensory gating within the human brain," Biol. Psychiatry, vol. 53, pp. 511-519, 2003.

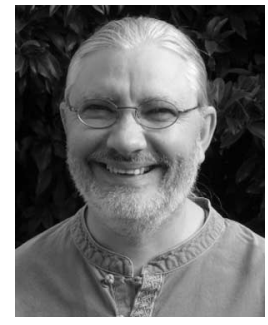

Patrick Celka received the Ph.D. degree from the Swiss Federal Institute of Technology, Lausanne, Switzerland, in 1995, in the fields of nonlinear electro-optical systems with application to CATV and chaos-based communication, chaos in time-delayed nonlinear systems and $1 \mathrm{D}$ chaotic maps.

He was with the Swiss Federal Institute of Technology during 1990-1998, where he was engaged in research on nonlinear circuits and systems and nonlinear dynamic systems and signals, and where he was earlier teaching at Associate Lecturer level, and later, became Senior Research Assistant. During 1999-2001, he was a Postdoctoral Fellow at the Queensland University of Technology, Brisbane, Australia From 2001 to 2004, he was with an R\&D company in Switzerland. He was a Senior Lecturer at the School of Engineering, Griffith University, Queensland, Australia. Since 2004, he has been with the School of Psychology, Griffith University, where he was a Full Member of the Applied Cognitive Neuroscience Research Centre. He is currently with the Lama Tzong Khapa Institute, Pomaia, Italy. His current research interests include nonlinear dynamical systems theory, nonlinear signal and system modeling and identification, chaos theory and its application, nonlinear adaptive algorithms, biomedical engineering, and wholistic models of the mind/body system.

Dr. Celka is the Founder of PeerReview (2008) Signals and Systems, an e-Service for academics and researchers in signals and systems theory and applications.

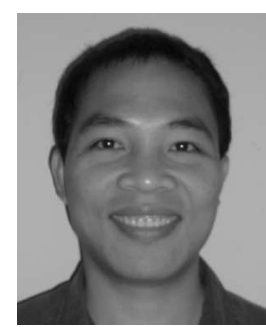

Khoa N. Le (M'03) received the Ph.D. degree in electrical and computer systems engineering from Monash University, Melbourne, Australia, in 2002

From 2002 to 2003, he was a Research Associate in the Department of Electrical and Computer Systems Engineering, Monash University, where he was engaged in research on signal processing and telecommunications. He is currently a Lecturer at the School of Engineering, Griffith University, Queensland, Australia. His current research interests include image processing, chaos, wavelets with applications, and wireless communications.

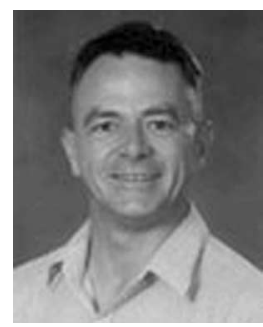

Timothy R. H. Cutmore received the M.Sc. degree in computer science in 1989 and the Ph.D. degree in psychology in 1987 both from Queens University, Kingston, ON, Canada.

During 1990, he was with the Department of Computer Science, York University Toronto, ON, teaching at the level of Assistant Professor. He is currently a Senior Lecturer in the School of Psychology, Griffith University, Brisbane, Australia. He is a regular Reviewer for the International Journal of Human Computer Studies, Clinical Neurophysiology, and Signal Processing. His current research interests include prototype portable sensors systems such as ECG and spirometry.

Dr. Cutmore was in 1999 named Teacher of the Year, and, in 2000, Supervisor of the Year in the School of Applied Psychology at Griffith University. 